\title{
A PHOTOMETRIC SEARCH FOR '5-MIN' OSCILLATIONS IN THE COMPONENTS OF ALPHA CENTAURI AND TWO OTHER DOUBLE STARS
}

\author{
L. A. Balona and $F$. Marang \\ South African Astronomical Observatory \\ P.0. Box 9 \\ Observatory 7935, Cape \\ South Africa
}

\section{INTRODUCTION}

The discovery of global solar 5-min oscillations has prompted the search for such oscillations in other stars. The main problem in detecting these variations is their expected small amplitude (in the sun it is less than $1 \mathrm{~m} / \mathrm{s}$ in velocity and $10^{-6}$ in integrated light). Two recent studies by Fossat et al. (1984) and Noyes et al. (1984) have suggested that the $\overline{5-m i n}$ oscillations in $\alpha$ Cen and $\varepsilon$ Eri may have been detected.

We attempted to detect these oscillations by means of conventional broad-band photometry in the components of three double star systems ( $\alpha$ Cen, HR4677 and HR5568). We selected these stars because they cover a wide range of spectral types ( $F 2 V$ to M2V). It was hoped that there may be a region where the pulsation amplitudes are particularly large. By using one component of a double star system to correct for transparency variations in the other, we also expected to obtain a larger signal-to-noise ratio.

\section{OBSERVATIONS}

The stars were observed with the $0.5-\mathrm{m}$ reflector of the SAAO using the Johnson B filter (with a heavy neutral density filter in the case of $\alpha$ Cen). All three systems have separations close to 20 arcsec. The observations were made on nights of good seeing using a diaphragm of 20 arcsec diameter. Contamination from the other component was not a problem. An integration time of 20 sec was used, allowing repeated alternate observations of each component every 75 seconds on average. As can be seen from Table 1, a minimum of four near-consecutive nights was obtained for each system. 
TABLE 1

Star

MK Type J.D. (2446500+) followed by hours observed in brackets

$\begin{array}{lll}\alpha \text { Cen A } & \text { G2V } & 16(7.0), 17(10.0), 21(6.9), 22(10.0), \\ \alpha \text { Cen B } & \text { K1V } & 23(2.3), 26(3.4), 27(2.0) . \\ \text { HR4677A } & \text { F2V } & 36(6.9), 37(1.9), 38(6.6), 41(4.3), \\ \text { HR4677B } & \text { F 3V } & 42(4.6) . \\ \text { HR5568A } & \text { K4V } & 23(4.1), 24(5.5), 26(5.8), 27(8.4) . \\ \text { HR5568B } & \text { M2V } & \end{array}$

\section{RESULTS}

After removing the trend due to transparency variations (as determined from one component of each double star system), we constructed a periodogram of the data. We found that an oscillation of $0.3 \mathrm{millimags}$ amplitude was detectable. If we merely removed the long-term trend due to transparency variations without using the companion to calibrate the trend, this figure was hardly increased. This suggests that transparency changes occured at a faster rate than the rate of observations, destroying the coherence expected from the close spatial separation.

We examined the periodograms of all six stars using various criteria to isolate variations of significant amplitude. These included constructing the autocorrelation of the periodogram and the power spectrum of the periodogram as suggested by Noyes et al. (1984) and Fossat et al. (1984). We concluded that these tests would probably have detected solar-type oscillations with an amplitude of about 0.1 millimags if these were present. We conclude that if solartype oscillations are present in main sequence stars, they are not likely to be discovered by conventional broad-band photometry.

\section{REFERENCES}

Fossat, E., Gelly, B. \& Grec, C., 1984. Theoretical problems in Stellar Stability and Oscillations, 229 .

Noyes, R.W., Baliunas, S.L., Belserene, E., Duncan, D.K., Horne, J. \& Widrow, L., 1984. Astrophys. J., 285, L23. 